(QMVC). Handgrip strength and six minute walk distance (SMWD) were also recorded.

Results As expected there were differences in BMI between the groups. There were also significant differences in muscle strength and $\mathrm{RF}_{\mathrm{CSA}}$ when corrected for body weight between $\mathrm{HC}$ and OSA groups and between SO and OSA groups, but no differences between HC and SO groups (Table 1). The SO group demonstrated higher measurements of strength and $\mathrm{RF}_{\mathrm{CSA}}$ than the HC group, however, the OSA group had lower measurements than both the HC and SO group. This translated to a functional difference as measured by the SMWD, again demonstrating the longest distance in the SO group and shortest in the OSA group.

Discussion This study has demonstrated that in those with a BMI $\geq 25 \mathrm{~kg} / \mathrm{m}^{2}$ there appears to be a beneficial effect of excess weight on peripheral muscle size, strength and function; this may be due to the extra load carried by these individuals exerting a training effect on the muscles. However, in those who are obese with SDB, the SDB seems to exert a negative effect on muscle size, strength and function which may be a result of the inflammation and hypoxia SDB can cause.

\section{P214 UTILISATION OF CARDIO-PULMONARY EXERCISE TESTING (CPET) AT AN ENGLISH ACUTE HOSPITAL}

E Parkes, VC Moore, D Comer, F Rauf, N Santana-Vaz, R Mukherjee. Birmingham Heartlands Hospital, Birmingham, UK

\subsection{6/thoraxjnl-2014-206260.343}

Background CPET has been extensively used in the pre-operative (general anaesthesia) risk stratification. More recently, the utility of CPET has become more defined in the evaluation of unexplained dyspnoea and in prognosticating pulmonary hypertension in a rational manner which is also less invasive for patients. We set out to evaluate the utilisation pattern of CPET within a 709-bedded central England acute hospital Trust spread across 3 sites in the second year of the establishment of the service.

Methods The source of referral (and reason) for CPET were retrospectively recorded and analysed between 01 July 2013 and 31 May 2014 (ten months).

Results The total number of CPET referrals received was 178 out of which 150 (84\%) were from surgical disciplines and 28
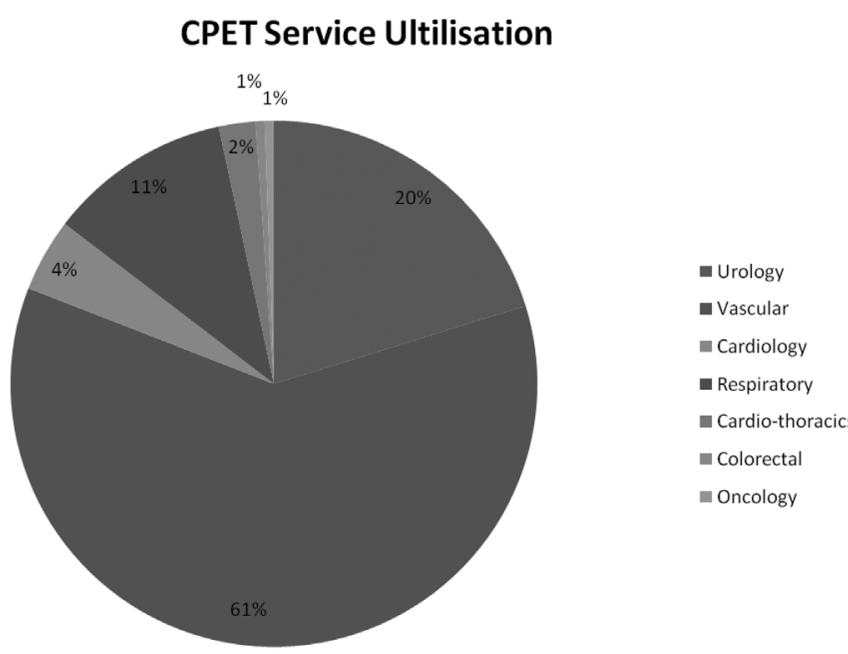

Abstract P214 Figure 1 CPET Service Utilisation
(16\%) from medical disciplines. Vascular surgery submitted made the majority of referrals $(108,61 \%)$ followed by colorectal surgery [see Figure]. Respiratory Medicine was the source of $11 \%$ of all referrals and Cardiology the source of $4 \%$.

Conclusions The dominant utilisation of CPET by vascular surgery is expected, given the NHS evidence adoption centre and National Institute for Health and Care Excellence (NICE) 2009 recommendations on risk-stratification for Abdominal Aortic Aneurysm surgery mortality. However, CPET offers a unique assessment tool for the investigation of patients with unexplained dyspnoea and has a potential to pre-empt invasive, unnecessary and expensive assessment without definitive diagnosis [Thing JER, Mukherjee B, Murphy K et al. Thorax 2011; 66 (4): A144]. It appears that a lot of work needs to be done among the UK general respiratory and cardiology/heart failure communities to promote the awareness, understanding and utilisation of CPET.

\section{Diagnostic and therapeutic interventional procedures}

\section{P215 REFERRAL PATTERNS FOR MEDIASTINAL STAGING WITH EBUS ACROSS A LUNG CANCER NETWORK A REPORT FROM THE MANCHESTER CANCER EBUS SUB-GROUP}

${ }^{1} \mathrm{M}$ Evison, ${ }^{1} \mathrm{P}$ Crosbie, ${ }^{1} \mathrm{~J}$ Morris, ${ }^{1} \mathrm{~J}$ Martin, ${ }^{1} \mathrm{R}$ Shah, ${ }^{1} \mathrm{H}$ Doran, ${ }^{2} \mathrm{~J}$ Hoyle, ${ }^{3} \mathrm{~S}$ Bailey, ${ }^{3} \mathrm{D}$ Rana, ${ }^{4} \mathrm{R}$ Sundar, 'R Booton. 'University Hospital South Manchester, Manchester, UK, ${ }^{2}$ Peninne Acute NHS Trust, Manchester, UK; ${ }^{3}$ Central Manchester NHS Foundation Trust, Manchester, UK; ${ }^{4}$ Wrightington, Wigan and Leigh NHS Foundation Trust, Manchester, UK

\subsection{6/thoraxjnl-2014-206260.344}

Introduction Manchester Cancer is a large Cancer Network in the North West of England, consisting of ten NHS Trusts. There is known variability in lung cancer outcomes across the ten trusts, including resection rates. We have examined the referral patterns of each individual trust for EBUS mediastinal staging and compared this with the trust's lung cancer resection rates.

Methods The Manchester Cancer EBUS sub-group has data for all EBUS referrals from each of the ten NHS trusts in our Network in 2012. The National Lung Cancer Audit Report 2013 provides the number of lung cancer patients diagnosed at each

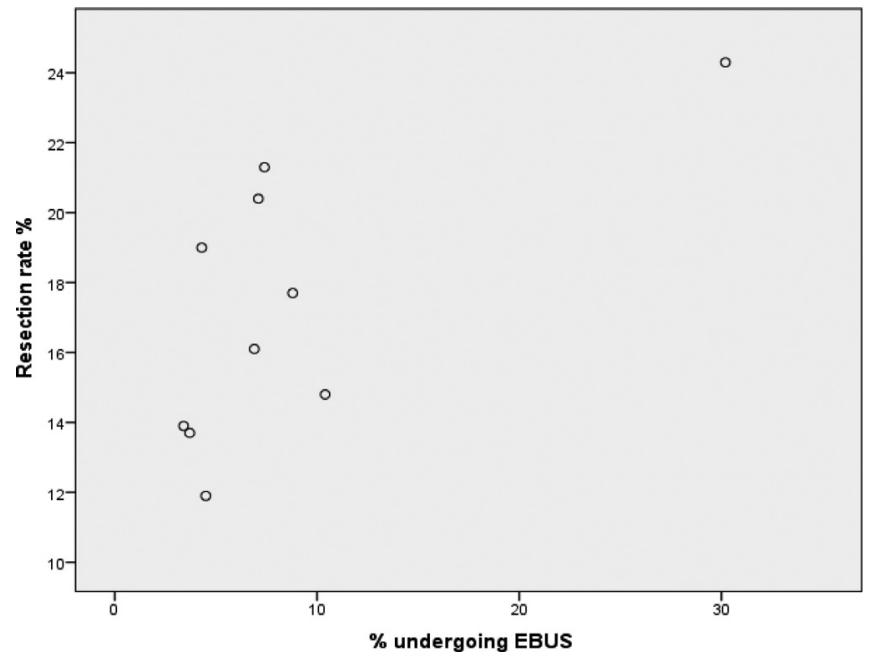

Abstract P215 Figure 1 
trust for the same time period. From this data we could estimate the proportion of lung cancer patients from each trust that were referred for mediastinal staging with EBUS.

Results In 2012, 2302 patients were diagnosed with lung cancer in this Network. In the same period, 193 patients were referred for EBUS mediastinal staging (8.4\%). The proportion of lung cancer patients referred for mediastinal staging with EBUS varied significantly across the ten trusts ranging from $3.4 \%$ to $30.2 \%$ $(\mathrm{p}<0.0001)$. The spearman co-efficient was $0.60(\mathrm{p}=0.07)$ suggesting a possible relationship between the proportion of patients referred for EBUS and surgical resection (Figure 1). However, this may be due to a very high rate of staging EBUS at one trust, which if excluded yields a spearman co-efficient of $0.45(\mathrm{p}=0.22)$.

Discussion It is highly concerning that only $8 \%$ of lung cancer patients underwent EBUS nodal staging in our network given $52 \%$ of UK patients with histologically confirmed NSCLC are stage I-III at the time of diagnosis. It is of note that the Trust with the highest proportion of patients undergoing EBUS nodal staging have the highest surgical resection rate and three of the four Trusts with the lowest resection rates refer $<5 \%$ of patients for EBUS nodal staging. Standardisation of referral practice across the Network is a key future goal for the EBUS Sub-group.

\section{P216 LOCAL ENDOBRONCHIAL ULTRASOUND (EBUS) SERVICE REDUCES WAITING TIME FOR TEST RESULTS}

JL Dickson, M Lawson. Broomfield Hospital, MEHT, Chelmsford, UK

\subsection{6/thoraxjn-2014-206260.345}

Introduction Endobronchial ultrasound (EBUS) is increasingly used in investigating mediastinal lymphadenopathy. A recent Thorax paper suggested EBUS should only be performed in large centres to ensure quality. A recently established EBUS service at Broomfield Hospital appeared to reduce the time taken to obtain results compared to the regional service previously used. The service was audited to ensure quality and evaluate changes to pathway times.

Objectives Compare time from decision for EBUS to test result between local and regional service and ensure safety and accuracy.

Methods Data were collected prospectively for all EBUS cases after the local service was established in August 2013. Accuracy and safety of the service were audited based on the first 8.5 months of operation. Data were extracted from the MDT database for patients referred to the regional service between November 2013 and January 2014. Time from MDT decision for EBUS referral and subsequent MDT discussion of results were compared between both services. Data were compared using the Mann-Whitney $\mathrm{U}$ test using the statistics package in R. Results Average time from decision to EBUS result was 19 days in the local service based on the first 21 cases performed. There was a 40 day average turnaround for the regional service based on the 10 cases referred between November 2013 and January 2014. This represented a statistically significant reduction in waiting time of 21 days $(\mathrm{p}=0.0001)$. The local service was safe and accurate with no reported complications in 42 cases over the first 8.5 months and an overall accuracy of $88 \%$ increasing to $94 \%(31 / 33)$ in suspected cancer cases.

Conclusions Our recently established local EBUS service is safe and accurate but also significantly reduces the time between decision to EBUS and test results discussed in MDT compared to the regional referral service previously used. This is important for patients on the 62-day cancer pathway. A local service enables people to undergo investigations nearer to home. The establishment of national reference standards for EBUS is important to ensure optimal quality but this can be achieved in local services which may offer additional benefits to patients.

\section{P217 THE NEGATIVE PREDICTIVE VALUE OF ENDOSONOGRAPHY FOR MEDIASTINAL STAGING OF NON-SMALL CELL LUNG CANCER}

${ }^{1} \mathrm{~K}$ Sayal, ${ }^{2} \mathrm{M}$ Scarci, ${ }^{3} \mathrm{~N}$ Carroll, ${ }^{1} \mathrm{~B}$ Dougherty. ${ }^{1}$ Department of Thoracic Oncology, Papworth Hospital NHS Foundation Trust, Papworth Everard, Cambridge, UK; ${ }^{2}$ Department of Thoracic Surgery, Papworth Hospital NHS Foundation Trust, Papworth Everard, Cambridge, UK; ${ }^{3}$ Department of Radiology, Cambridge University NHS Foundation Trust, Cambridge, UK

\subsection{6/thoraxjnl-2014-206260.346}

Introduction and objectives The ASTER trial demonstrated that the sensitivity of combined endobronchial/endoscopic ultrasound [EBUS/EUS] is similar to that of mediastinoscopy. ${ }^{1}$ NICE guidelines now recommend combined EBUS/EUS for initial mediastinal staging as an alternative to surgical staging. ${ }^{2}$ Although surgical staging following negative endosonography is recommended, ASTER showed that 11 mediastinoscopies are required to identify one case of mediastinal disease. We aimed to determine the negative predictive value of EBUS/EUS for mediastinal staging in our practice.

Method We identified all patients who had undergone primary surgical resection with lymph node sampling for NSCLC between May 2012 and April 2014 previously staged with CT, PET-CT, EBUS+/-EUS and/or mediastinoscopy. Demographic,

\begin{tabular}{lll} 
Abstract P217 Table 1 & Patient characteristics \\
\hline & Number & Percentage (\%)(\% \\
\hline Median age & 68 years & \\
Range & $43-87$ years & \\
Histology: & & \\
Squamous & 19 & 45 \\
Adenocarcinoma & 19 & 45 \\
Other & 4 & 10 \\
EBUS only staging & 35 & 83 \\
EUS only staging & 1 & 2 \\
Combined EBUS/EUS staging & 6 & 14 \\
Pre-EBUS/EUS PET/CT staging: & & \\
T1 & 10 & 24 \\
T2 & 23 & 55 \\
T3 & 7 & 17 \\
T4 & 2 & 5 \\
Pre-EBUS/EUS PET/CT staging: & & \\
N0 & 9 & 21 \\
N1 & 13 & 31 \\
N2 & 10 & 24 \\
N3 & 6 & 14 \\
Equivocal & 4 & 10 \\
Tumour localization: & 13 & 26 \\
Left lower lobe & 11 & \\
Left upper lobe & & \\
Right upper lobe & & \\
Right middle lobe & & \\
Right lower lobe & & \\
\hline & 11 & \\
\hline & & \\
\hline
\end{tabular}

